\title{
Comparison of the performance of Skin Prick and ISAC Tests in the diagnosis of allergy
}

\author{
${ }^{1}$ Experimental Allergy Unit, IDI-IRCCS, Rome, Italy \\ ${ }^{2}$ Immunology and Allergology SSD, Ospedale S. Maria degli Angeli, Pordenone, Italy \\ ${ }^{3}$ Clinic Patology U.O.C., Ospedale Buccheri La Ferla F.B.F., Palermo, Italy \\ ${ }^{4}$ Allergology and Clinical Immunology SOS, USL Toscana Centro, Prato, Italy
}

\section{KEY WORDS}

Skin Prick test; microarray test; allergic disease; dermatological disease; food allergy; singleplex; multiplex.

\author{
Corresponding author \\ Enrico Scala \\ Clinical Allergy Unit \\ Fondazione Luigi Maria Monti - IDI-IRCCS \\ Via dei Monti di Creta 104 \\ 00167 Rome, Italy \\ E-mail: e.scala@idi.it
}

Doi

10.23822/EurAnnACI.1764-1489.135

\begin{abstract}
Summary
The recent European Union and Italian regulations in the matter of in vivo test could strongly impact on current diagnostic approach, increasing the usage of in vitro tests in daily clinical practice.

We evaluated 506 patients with both skin prick test and a microarray system (ImmunoCAP ISAC 112). The overall evaluation between ImmunoCAP® ISAC vs SPT showed a moderate agreement ( $k=0.509$, 95\% C.I. $0.480-0.540$, SE: 0.016) considering both aeroallergens and food allergens. When we considered the concordant results (double-positive plus double-negatives), the agreement ranged from 69\% to $80 \%$ for pollen allergens, between $74 \%$ and $76 \%$ for dust mites, and between $74 \%$ and $93 \%$ for animal epithelia. In the case of food allergens, the accordance was pretty lower, accounting values ranging from $67 \%$ to $86 \%$. ISAC testing identified from $22 \%$ to $26 \%$ more cases than SPTs in peach and nuts hyper-sensitivity. In 2.8\% of the control group, the ISAC-test failed to detect an allergy sensitization caused by dust mite, shrimp, Anisakis, or seed storage proteins.

Multiplex testing is more than a promising tool for more precise and comprehensive profiling of allergic patients and can be considered as a second-line approach, after the anamnesis, in the diagnosis of allergic diseases.
\end{abstract}

\section{Introduction}

In the classical inductive allergic diagnostic workup (Top-down approach), based on the patient-reported history, several tests can be performed to confirm or exclude possible causes of sensitization (1). Classically, the first line investigation is represented by extract-based skin testing, usually using a panel of biological sources, chosen following the current guidelines (2). Furthermore, in vitro singleplex tests with extract-based analytes are commonly prescribed as a sort of confirmatory evaluation of the in vivo testing and the single components are performed for an in-depth analysis. Several multiplex systems have been recently developed, allowing the evaluation of hundreds of distinct components at the same time and in the same patient. Such an in vitro test could detect a comprehensive profile of $\operatorname{IgE}$ sensitization (3). Due to higher costs, in most allergy units in Italy
ISAC test is offered to the patient as a private test and therefore is currently prescribed only in selected situations or in case of complex diagnoses (4).

In 2001 a directive (2001/83/EC) of the European Parliament stated that "[..] no medicinal product (including allergens for in vivo tests) may be placed on the market of a Member state unless a marketing authorization has been issued by the competent authorities [...]" (5). Recently this directive was implemented in Italy and several determinations have been published in the GAZZETTA UFFICIALE establishing the trading denial for many allergenic products for in vivo tests and immunotherapy in patients suffering from environmental or food allergies.

Given the likely downsizing soon of traditional in vivo in favor of a predominantly in vitro diagnostic assessment, we retrospectively evaluated a large cohort of patients to verify the amount 
of loss (or gain) in diagnosis precision obtained with a comprehensive proteomic approach instead of a classical multistep evaluation utilizing skin prick testing $(4,6,7)$.

\section{Materials and methods}

The observational controlled study cohort was enrolled at the outpatient Allergy Unit of IDI-IRCCS in Rome, a National Reference Center for Allergic and Dermatological diseases. Demographic details together with clinical data (food-related reactions, respiratory and dermatological symptoms) were recorded using the TD-Synergy ${ }^{\circledR}$ Laboratory Information System (Siemens Healthcare Diagnostics, Muenchen, Germany) and a customized electronic database.

The study received ethical approval from IDI-IRCCS Ethical Committee (496/1).

\section{Patients}

Patients aged 18 years and over, born in Central or Southern Italy presenting with a history of adverse reactions to foods, allergic rhinitis, bronchial asthma and/or atopic dermatitis were recruited between January and December 2019.

The case group consisted of 256 patients (males: 135, mean age $34 \pm 17$; range 18-69), having a clear reactivity to one or more biological sources currently spotted in the ImmunoCAP ISAC. Clinical categorization was as follows: [Food Allergy, FA] history of symptoms unequivocally suggestive of adverse reaction to a suspected plant food-derived trigger(s), including urticaria and external angioedema, laryngeal angioedema, respiratory difficulty and/or pre-syncope/syncope in the last 6 months; [Respiratory Symptoms, RS] symptoms of rhino-conjunctivitis and/or bronchial asthma only.

The control group comprised 250 adults (males: 115, mean age $33 \pm 16$; range 18-72) with negative results after the ImmunoCAP ISAC test, despite a patient reported a history of chronic urticaria $(64 \%)$, atopic dermatitis $(28 \%)$, or vasomotor rhinitis (19\%).

\section{Diagnostic assays}

\section{Skin Prick Tests}

All subjects underwent Skin Prick Tests (SPT) to a series of glycerinated aeroallergen and food extracts (either from Stallergenes, Antony, France or ALK Abelló, Horsholm, Denmark from), and positive and negative control solutions (histamine hydrochloride $10 \mathrm{mg} / \mathrm{mL}$ and diluent) on the volar forearms. The inhalant panel included pollen from a grasses mixture (Phleum pratense, Lolium perenne, Poa pratensis), Artemisia vulgaris, Parietaria judaica, Plantago lanceolata, olive, birch, hazel, oak, cypress, plane trees, Dermatophagoides pteronyssinus, and farinae, dog, cat, and horse dander, Alternaria alternata, Cladosporium herbarum, Aspergillus mixture, latex, and cockroach. The panel of food allergens, all available as extracts $1: 20 \mathrm{w} / \mathrm{v}$, included Anisakis simplex, shrimp, peanut, walnut, and hazelnut. Peach extract (ALK Abelló) was chosen as a marker for nsLTP sensitization and birch pollen as a marker for pollen food syndrome related to PR-10 proteins $(8,9)$. SPTs were performed using sterile stainless steel standardized lancets (Stallergenes) by the same operator, and taken at $15 \mathrm{~min}$, using standardized techniques according to international guidelines (10).

\section{Serum analysis}

A semi-quantitative allergen microarray assay was used to determine the individual participant's specific IgE sensitization to 112 allergen components in triplicate, measured using the Immuno Solid-phase Allergen Chip (ImmunoCAP ISAC 112) microarray system platform according to the manufacturer's instructions (Thermo Fisher Scientific, Uppsala, Sweden). Specific IgE values were expressed in ISAC standard units (ISU), with values of 0.3 ISU or greater considered positive.

For the specific purpose of comparing allergenic molecule $\operatorname{IgE}$ prevalence to extract based SPT evaluation, single molecular results from each distinct biological source or panallergen subset were pooled together as follow: grasses (Cyn d $1+\mathrm{Phl} \mathrm{p} 1+\mathrm{Phl}$ p $11+\mathrm{Phl}$ p $12+\mathrm{Phl}$ p $2+\mathrm{Phl}$ p $4+\mathrm{Phl}$ p $5.0204+\mathrm{Phl}$ p $6+$ Phl p 7); cypress (Cup a $1+$ Profilin + Polcalcin); mugwort (Art v $1+$ Art v $3+$ Profilin + Polcalcin); plane tree (Pla a 1.dic + Pla a 2.dic + Pla a 3.dic + Profilin + Polcalcin); birch tree (Betv $1+$ Bet v $2+$ Bet v 4); oak tree (Bet v $1+$ Bet v $2+$ Bet v 4); pellitory (Par j $2+$ Profilin + Polcalcin); olive tree (Ole e $1+$ Ole e $7+$ Ole e $9+$ Profilin + Polcalcin); Dermatophagoides pteronyssinus (Der p $1+$ Der p $10+$ Der p $2+$ Blot $5+$ Pen m 2); Dermatophagoides farinae (Der f $1+$ Der f $2+$ Der p $10+$ Blo $5+$ Pen m 2); Alternaria (Alt a1 + Alt a 6); Aspergillus (Asp f1 + Asp f $3+$ Asp f 6); cat dander (Fel d $1+$ Fel d $2+$ Fel d 4); dog dander (Can f 1 + Can f $2+$ Can f $3+$ Can f 5); horse (Equ c $1+$ Equ c 3); latex (Hev b $1+$ Hev b $3+$ Hev b $5+$ Hev b $6.01+$ Hev b 8.0204); Blattella (Bla g $1+$ Bla g $2+$ Bla g $5+$ Bla g 7); Anisakis (Ani s $1+$ Ani s 3); peach (Pru p $1+$ Pru p 3); shrimp (Pen m $1+$ Pen $\mathrm{m} 2+$ Pen $\mathrm{m} 4)$; hazelnut (Cor a 1.0101 + Cor a $1.0401+$ Cor a $8+$ Cor a 9); peanut (Ara h $1+$ Ara h $2+$ Ara h $3+$ Ara h $6+$ Ara h $8+$ Ara h $9+$ Profilin); walnut (Jug r $1+$ Jug r $2+$ Jug r 3); LTP (Ara h $9+$ Art v $3+$ Cor a $8+$ Jug r $3+$ Ole e $7+$ Pla a $3+$ Pru p $3+$ Tri a 14); Profilin (Bet v $2+$ Hev b $8.0204+$ Mer a $1+$ Phl p 12); Polcalcin (Bet v $4+$ Phl p 7$)$.

\section{Statistical analysis}

All data were analyzed using the SPSS/PC + statistical package for statistical evaluation (SPSS, version 15, Chicago, IL). The TD-Synergy Laboratory Information System was used to search 
and collect demographic (age and gender), clinical and laboratory data for Allergy Clinic patients who attended the outpatient Allergy clinic and underwent specific IgE testing.

Each variable of interest obtained with SPTs or the microarray system was dichotomized (as negative or positive), and the degree of relationship between the categorical variables studied was analyzed using the Pearsons' $\chi^{2}$ or Fisher's exact test when indicated.

Inter-rater agreement between SPT and ImmunoCAP ISAC was calculated for qualitative outcomes (positive-negative); Cohen's kappa coefficient $(\mathrm{k})$, positive and negative agreement were assessed for every single extract based on skin prick test (SPT) result and molecule considered. As conventionally assumed, kappa results have been interpreted as follows: $\mathrm{k}: \leq 0$ no agreement, 0.01-0.20 none to slight, 0.21-0.40 fair agreement, 0.41-0.60 moderate agreement, 0.61-0.80 substantial and 0.811.00 almost perfect agreement (11).

\section{Results}

\section{Skin prick tests}

Grass pollen allergens $(67,6 \%)$, house dust mites $(52,9 \%)$, and cypress pollen allergens $(52,8 \%)$ were the top-ranking reactivity to aeroallergens recorded in the case group. Peanut $(29,8 \%)$, peach $(17,6 \%)$, and walnut $(16,4 \%)$ represented the most frequently positive result in food SPT evaluation.

Respiratory symptoms were significantly associated with hyper-reactivity to SPTs $v$ s birch pollen ( $\mathrm{p}=0,02$; OR=2,67; 95\% $\mathrm{CI}=1,13-6,30)$, grasses $(\mathrm{p}=0,01 ; \mathrm{OR}=2,24 ; 95 \% \mathrm{CI}=1,20$ $4,19)$, cypress tree $(\mathrm{p}=0,02 ; \mathrm{OR}=2,04 ; 95 \% \mathrm{CI}=1,10-3,81)$, and plane-tree $R(p=0,03 ; O R=2,56 ; 95 \% C I=1,02-6,41)$.
Oral allergy syndrome (OAS) occurrence was associated with a positive SPT to hazelnut ( $<<0.001 ; \mathrm{OR}=4,71 ; 95 \% \mathrm{CI}=2,28$ 9,75), birch ( $\mathrm{p}=0,001 ; \mathrm{OR}=3,04 ; 95 \% \mathrm{CI}=1,47-6,28)$, and mugwort pollen $(\mathrm{p}=0,001 ; \mathrm{OR}=3,06 ; 95 \% \mathrm{CI}=1,49-6,25)$. Mugwort reactivity was also linked to the occurrence of severe reaction $(S R)$ to food $(\mathrm{p}=0,01 ; \mathrm{OR}=2,18 ; 95 \% \mathrm{CI}=1,22-3,91)$. Seven out of 250 subjects in the control group, despite the negative results after the ISAC testing, had a positive SPT to dust mites in 3 cases $(1.4 \%)$, to shrimp in 2 patients $(0.9 \%)$, to Anisakis in one subject $(0.5 \%)$, and peanut in one participant $(0.5 \%)$ (figure 1 ).

\section{Microarray}

The ISAC results showed a profile of sensitization comparable to what recorded with STP. Grass pollen allergens $(56,6 \%)$, cypress molecules $(54,7 \%)$, and house dust mite $(48,4 \%)$ component reactivity were the most commonly observed among the inhalant molecules tested, whereas $39,3 \%$ of the food reactive patients had a positive test to peanut allergens, 38,3\% to peach components, and $31,2 \%$ to walnut molecules.

RS occurrence was strictly associated with ISAC test reactivity to molecules belonging to mugwort Art v 1 ( $\mathrm{p}=0,03$; OR=3,20; 95\% CI=1,09-9,38); cypress pollen Cry j 1 ( $\mathrm{p}=0,01$; OR=2,25; $95 \% \mathrm{CI}=1,16-4,35)$ and Cup a $1(\mathrm{p}<0,001 ; \mathrm{OR}=2,55 ; 95 \%$ $\mathrm{CI}=1,34-4,84)$, and grasses Cyn d 1 ( $\mathrm{p}<0,001 ; \mathrm{OR}=2,57$; 95\% CI $=1,35-4,90)$ and Phl p $1(\mathrm{p}<0,001 ; \mathrm{OR}=2,53 ; 95 \%$ $\mathrm{CI}=1,33-4,79)$.

Severe Reactions (SR) to food were strictly linked to molecular reactivity to the $2 S$ Albumin from Brazilian nut Ber e $1(\mathrm{p}=0,01$; $\mathrm{OR}=9,27 ; 95 \% \mathrm{CI}=1,12-76,48)$, nsLTPs from peanut Ara h 9 $(\mathrm{p}<0,001 ; \mathrm{OR}=5,79 ; 95 \% \mathrm{CI}=2,79-12,01) ;$ mugwort Art v 3

Figure 1 - Clinical data of patient in the control group (Urt: chronic spontaneous urticaria; R: Rynithis; AD: Atopic Dermatitis; GI: gastro-intestinal symptoms; BA: Bronchial Asthma; Pen m: Shrimp; Ani s: Anisakis simplex; SSP: Seed Storage protein; Der p: dust mite). The extract reactivity of patients not detected by ISAC test ("missing") is reported on the right.
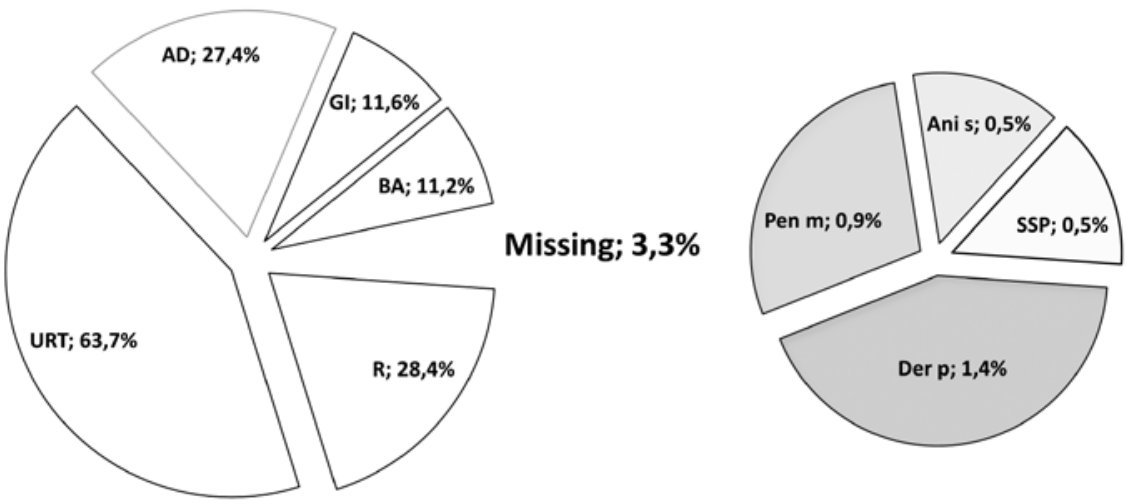
$(\mathrm{p}<0,001 ; \mathrm{OR}=3,38 ; 95 \% \mathrm{CI}=1,75-6,52)$; hazelnut Cor a 8 $(\mathrm{p}=0,00 ; \mathrm{OR}=4,49 ; 95 \% \mathrm{CI}=2,14-9,42)$; walnut Jug r 3 ( $\mathrm{p}<$ $0,001 ; \mathrm{OR}=4,34 ; 95 \% \mathrm{CI}=2,30-8,19)$; plane tree Pla a $3(\mathrm{p}<$ $0,001 ; \mathrm{OR}=4,18 ; 95 \% \mathrm{CI}=2,21-7,90)$; peach Pru $\mathrm{p} 3(\mathrm{p}<0,001$; $\mathrm{OR}=5,32 ; 95 \% \mathrm{CI}=2,89-9,80)$, and wheat Tri a $14(\mathrm{p}=0,03$; $\mathrm{OR}=2,71 ; 95 \% \mathrm{CI}=1,06-6,98)$. OAS was significantly associated with PR10 molecules from birch Bet v $1(\mathrm{p}=0,01 ; \mathrm{OR}=2,23$; 95\% CI=1,17-4,23); apple Mal d 1 ( $\mathrm{p}=0,01 ; \mathrm{OR}=2,52 ; 95 \%$ $\mathrm{CI}=1,26-5,03)$; peach Pru p $1 \quad(\mathrm{p}=0,03 ; \mathrm{OR}=2,15 ; 95 \%$ $\mathrm{CI}=1,06-4,36)$, and hazelnut Cor a $1.0401(\mathrm{p}=0,03 ; \mathrm{OR}=2,15$; $95 \% \mathrm{CI}=1,06-4,36$ ).

In addition, we verified SPT results in subjects with panallergen reactivity. As shown in figure 2, a significantly higher number of profilin reactive participants had a positive test to grasses, mugwort, birch, and hazel trees. Polcalcin sensitized individuals were more likely to be reactive to all kinds of pollen allergen, except for the olive tree. nsLTP hyper-sensitivity was associated with an increased occurrence of mugwort, plane tree, and pellitory STP reactivity. PR10 population showed an increased amount reactivity to birch, hazel and oak trees.

\section{SPT and Microarray comparison}

The comparison between SPT outcomes and ImmunoCAP ISAC evaluation is detailed in table $\mathbf{I}$. The overall evaluation between ImmunoCAP® ISAC vs SPT showed a moderate agreement $(\mathrm{k}=0.509,95 \%$ C.I. $0.480-0.540$, SE: 0.016) considering both aeroallergens and food allergens.

Among the inhalant allergens, no agreement $(\mathrm{k} \leq 0)$ was observed for cockroach and Aspergillus, slight agreement for latex $(\mathrm{k}=0,096)$, a fair agreement for oak tree $(\mathrm{k}=0,235)$, plane tree $(\mathrm{k}=0,321)$, dog dander $(\mathrm{k}=0,329)$, and pellitory $(\mathrm{k}=0,404)$, moderate agreement for grasses $(\mathrm{k}=0,410)$, horse $(\mathrm{k}=0,416)$, olive tree $(k=0,425)$, cypress $(k=0,436)$, house dust mite [Der $f$ $(\mathrm{k}=0,494)$ and Der $\mathrm{p}(\mathrm{k}=0,515)]$, birch tree $(\mathrm{k}=0,501)$ and mugwort $(k=0,549)$, whilst a substantial agreement was found only in the case of cat dander $(\mathrm{k}=0,613)$ and alternaria $(\mathrm{k}=0,761)$.

The overall agreement for food allergens resulted in slight to fair agreement comparing extract-based ST and molecular components. Particularly, no agreement was found for shrimp allergens, slight agreement for walnut $(\mathrm{k}=0,170)$, and fair agreement for hazelnut $(\mathrm{k}=0,229)$, peanut $(\mathrm{k}=0,238)$, peach $(\mathrm{k}=0,317)$, and Anisakis $(\mathrm{k}=0,380)$.

In figure 3 the prevalence of component recognition profiles, in SPT reactors and not, is shown. Interestingly patients SPT-positive to grasses, pellitory, and olive tree showed a significantly higher prevalence of Phl p 1, Par j 2 and Ole e 1 IgE recognition, respectively, than the patients SPT-negative. In the case of pellitory-of-the-wall, significantly higher occurrence of Polcalcin recognition was achieved in SPT reactors, whilst patients with negative to skin testing showed a higher occurrence of Profilin recognition.

\section{Discussion}

Our data indicate that, in the majority of cases, only a moderate concordance among SPT and ISAC-test was found, whereas, in the case of food allergens, the concordance was even lower.

When we considered the concordant results (double-positive plus double-negatives), the agreement ranged from $69 \%$ to

Figure 2 - Prevalence of sensitization to pollen, as evaluated by means of SPT, in panallergen reactors. ${ }^{*} p 0.01$.

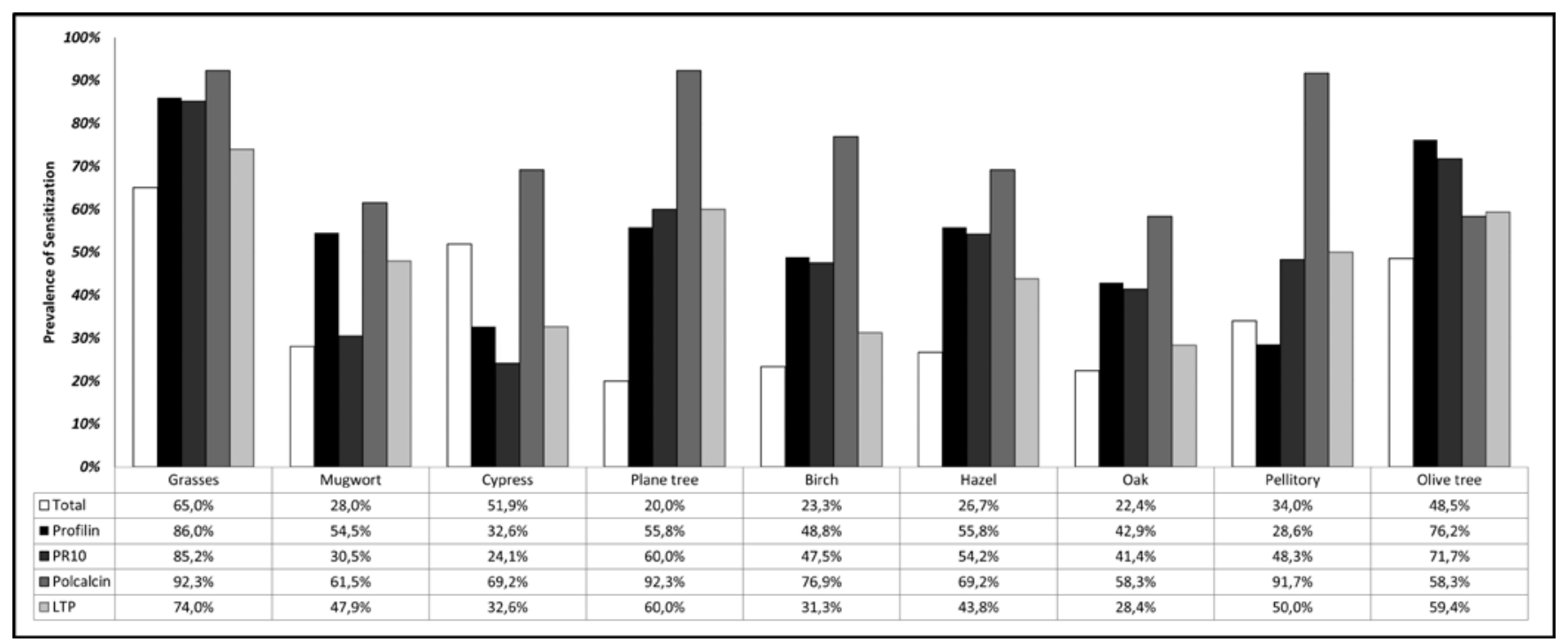


Table I - ImmunoCAP ISAC® vs skin prick test frequency reactivity comparison.

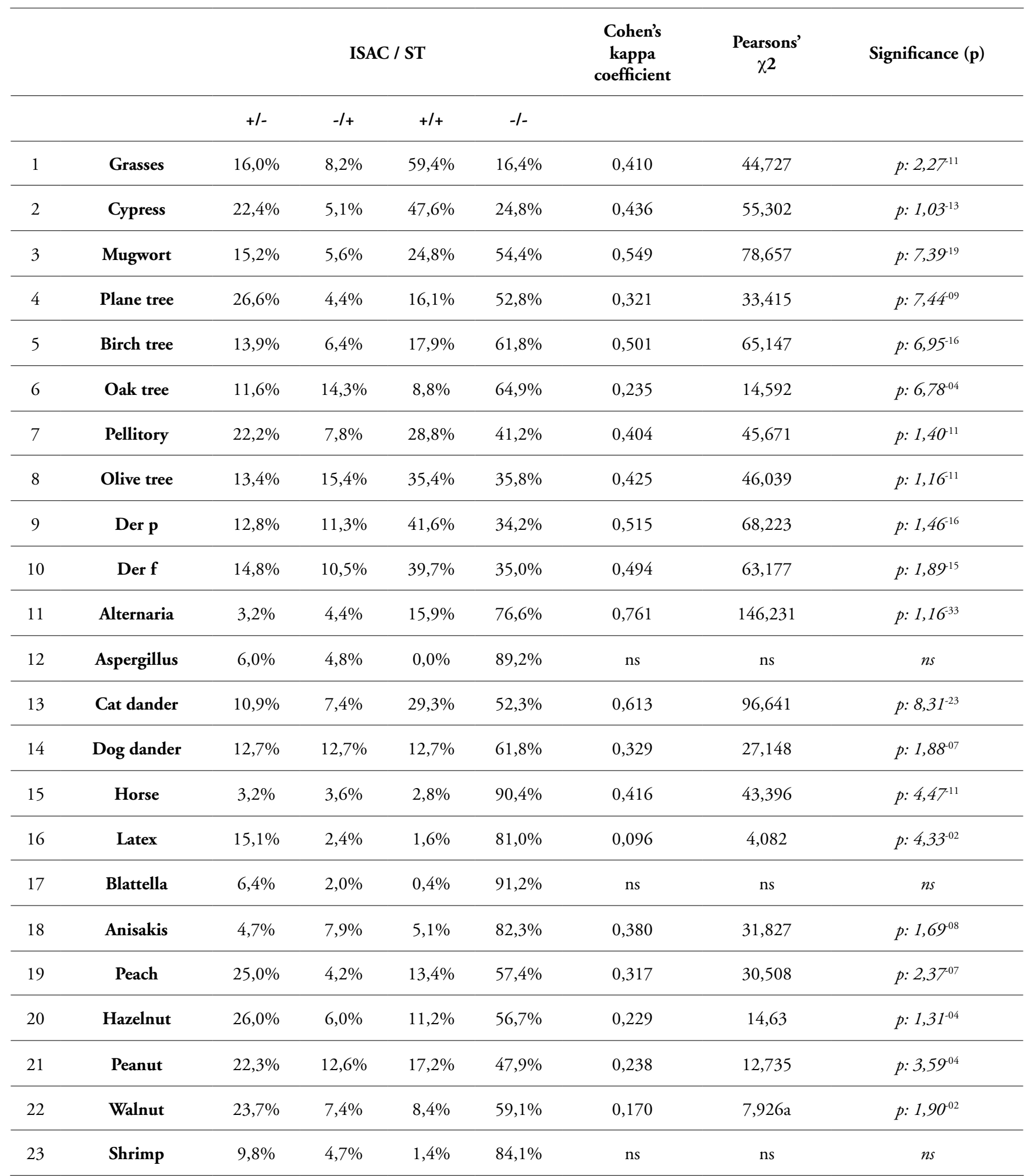


Figure 3 - Prevalence (\%) of component recognition profiles in patients detected (white bar), and not detected by skin prick test (grey bar) for pollen allergen (A), dust mite, mould, and animal dander extracts (B), and food allergen (C). ${ }^{*} p<0.01$.

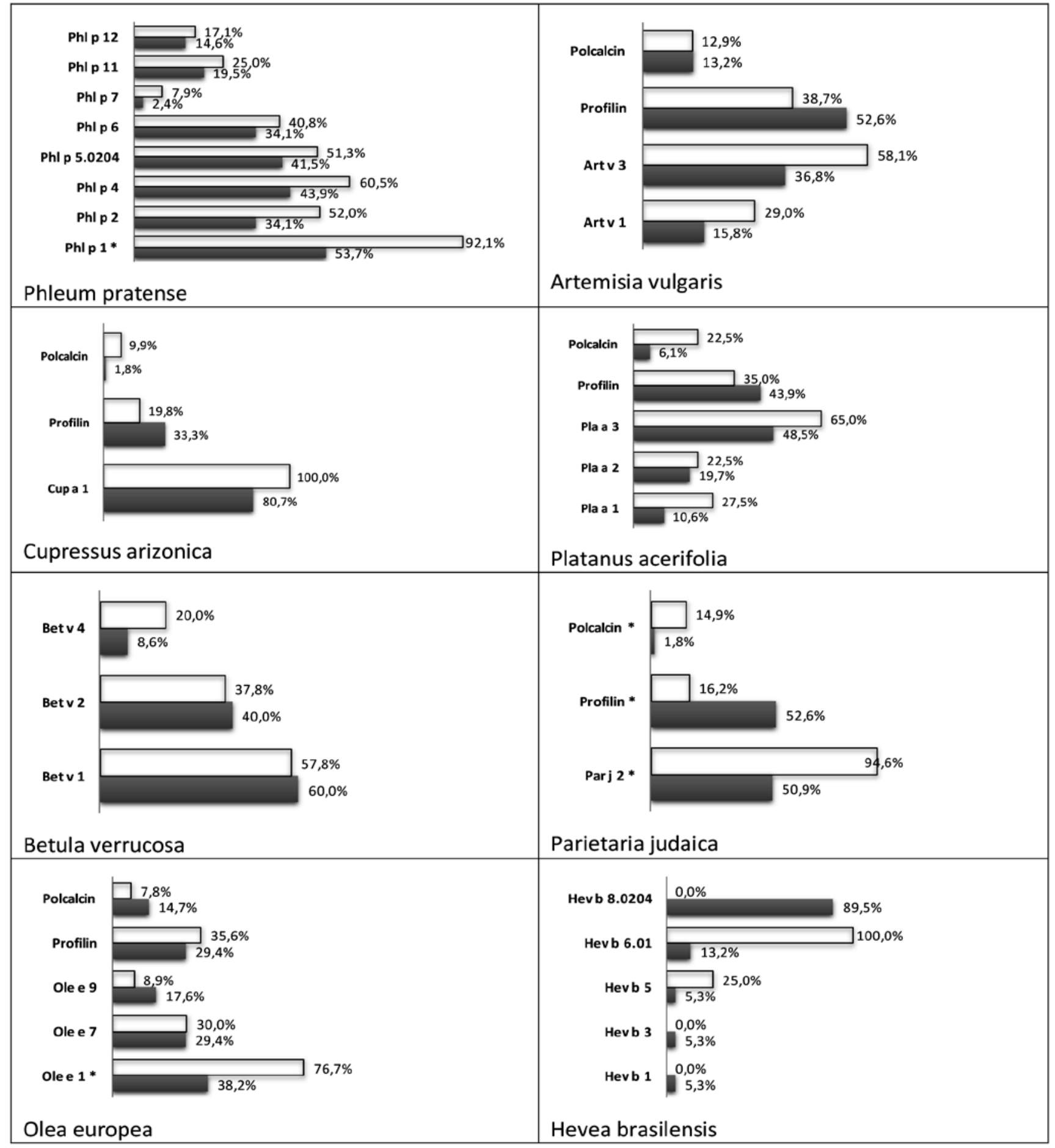

$\square$ SPT positive $\mathrm{SPT}$ negative $\left({ }^{*} \mathrm{p}<0.01\right)$ 


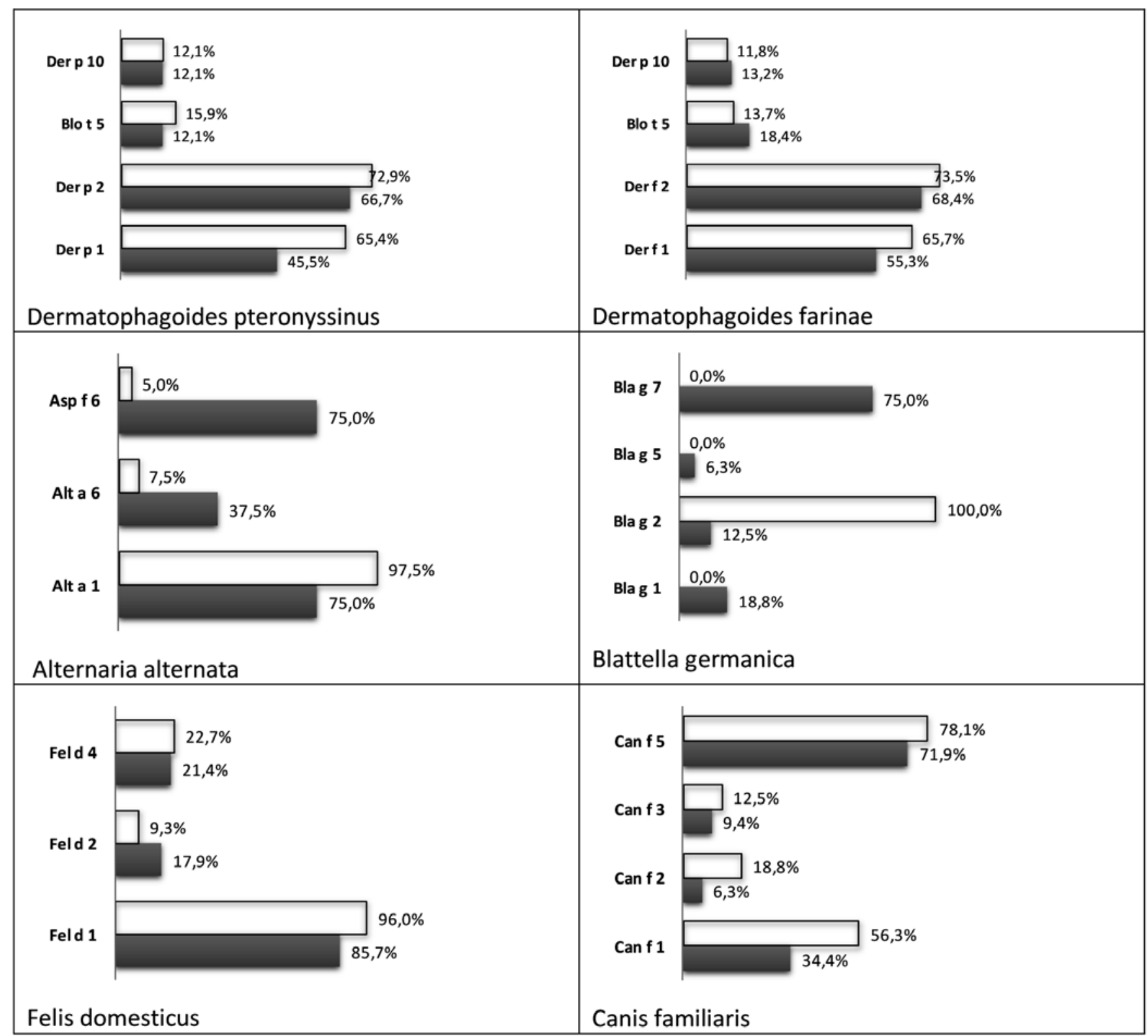

$\square$ SPT positive $\square$ SPT negative 


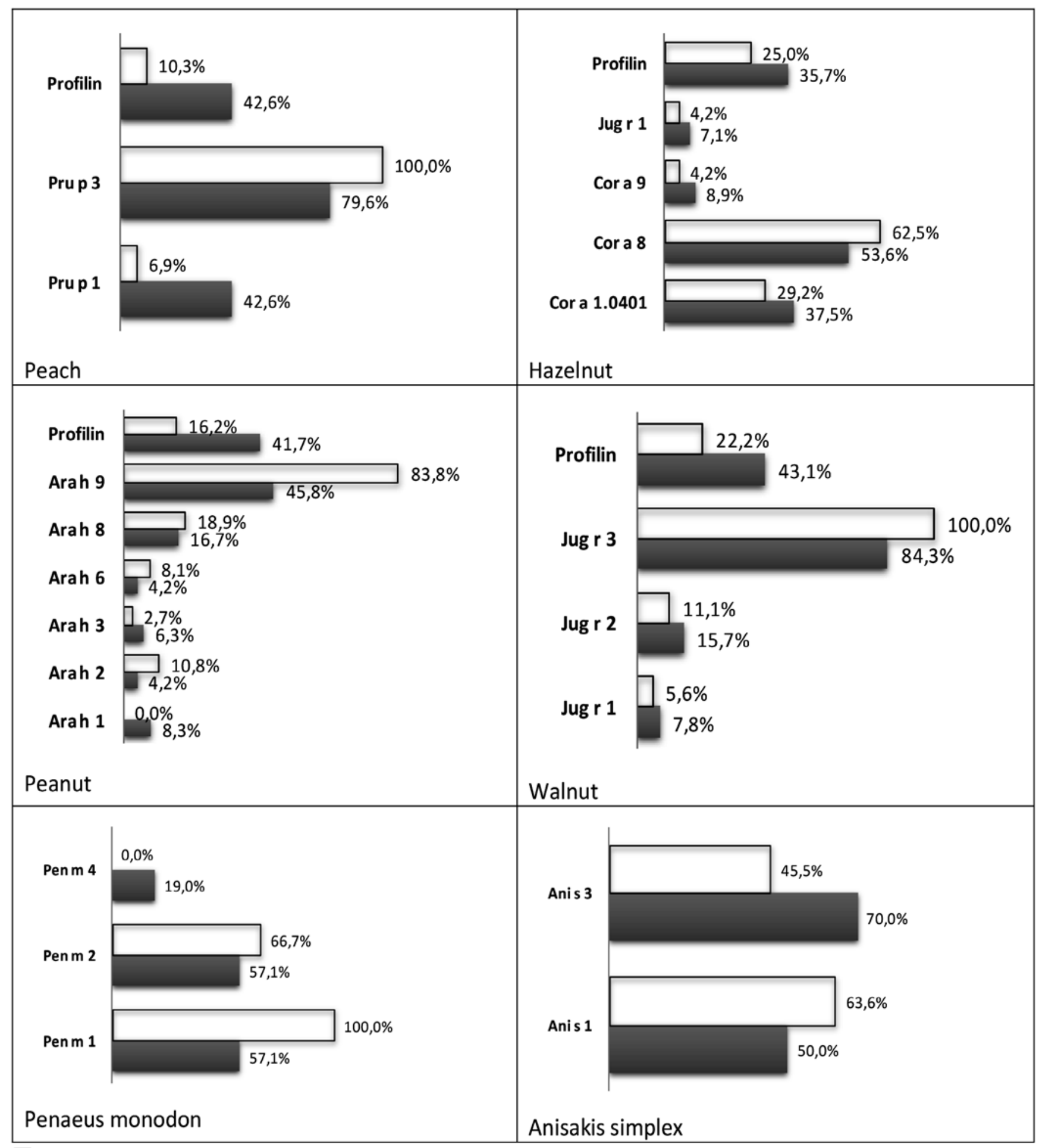


$80 \%$ for pollen allergens, between $74 \%$ and $76 \%$ for dust mites, and between $74 \%$ and $93 \%$ for animal epithelia. Surprisingly, the highest level of concordance was achieved by mould extracts (range from $89 \%$ to $92 \%$ ), probably due to the elevated frequency of double negative results.

As reported in table I, the detection rate of ISAC is frequently higher than SPTs. Overall, in the assessment of the reactivity to pollen allergens, ISAC identified about $10 \%$ more cases than SPT. It is worth noting that the higher detection rates by ISAC were observed in the case of plane-tree (22\%), cypress tree (17,3\%) and Parietaria (14.4\%). Previous studies have shown that certain pollen extracts such as pellitory and cypress tree lack Profilin either because this allergens is scarce in these sources or because it is a different isoform (12), and this could be the reason why several patients are better recognized by a specific molecular approach than after the usage of extracts possibly lacking relevant components. In the past, the usage of a pollen Profilin-enriched extract could overcome this caveat, but unfortunately, such a device is no more available in the market, due to the well known regulatory restriction about the usage of such product. Riccardo Asero et al. demonstrated that pollen extracts could significantly inhibit IgE reactivity to $\mathrm{rBet} \mathrm{v} 4$, whilst only grass pollen extract could inhibit $\mathrm{rPhl} \mathrm{p} 7 \mathrm{IgE}$ reactivity, as a further demonstration of the importance of a molecular approach for a better patient evaluation (13).

A similar result has been obtained in previous studies. Singleplex and multiplex systems showed comparable specificity and sensitivity in detecting grass and cypress pollen hyper-reactivity (14), or pollen (grass and birch) and animal dander (cat) allergy (15). In our cohort, a moderate agreement was found between SPT and ISAC for all these biological sources and a higher agreement for cat dander sensitization recognition.

In the case of food allergens, the accordance was pretty lower, accounting values ranging from $67 \%$ to $71 \%$. ISAC testing identified from $22 \%$ to $26 \%$ more cases than SPTs in peach and nuts hyper-sensitivity, in partial disagreement with previous studies where SPT and ISAC tests showed comparable results in the detection of patients with allergy to nuts (16).

On the other hand, it has been suggested that ISAC test can help in about $20 \%$ of cases, to identify the culprit allergen responsible for "idiopathic" anaphylaxis, particularly when the patient-reported history, SPT, and singleplex tests have not revealed the cause the adverse reaction (17). However, it is important to underline that, whatever the method, the presence of an IgE sensitization is only evidence of sensitization that should be correlated with the clinical history before drawing any conclusion.

Panallergen reactivity affected SPT outcome. Interestingly plane tree (18) and mugwort (19) sensitization were strictly related to FA and not to RS in the study group, as previously suggested (20). It is worthy of note that other mugwort pollen allergens, not fully identified yet, other than Art v 3 may be relevant as a food allergen, such as a $60 \mathrm{kDa}$ molecule isolated in mugwort extract, highly homologous to the fennel Api g 5 (21). As expected, Polcalcin recognition was associated with increased occurrence of pollen reactivity, and PR10 reactivity with positive SPT to trees belonging to the Fagales order $(8,22)$.

In $2.8 \%$ of the control group, the ISAC-test failed to detect a food allergy sensitisation caused by dust mite, shrimp, Anisakis, or seed storage proteins. It is widely known that in the case of house dust mite, the ISAC system could evaluate only molecules belonging to group $-1,-5,-10$ and, indirectly measured by Pen m 2 (23), group -20, whereas there are several other allergens not included in the ISAC platform, such as Der p 5, Der p 7 , Der p 11, and Der p 23 (24). Der p 23 was recently added in the latest version of ISAC (ISAC 122e) $(25,26)$.

On the other hand, it is extremely important to check Der $\mathrm{p}$ 1 or Der $\mathrm{p} 2$ reactivity in dust mite-positive patients before to prescribe allergen-specific immunotherapy, since it has been demonstrated that only this subset of patients seems to respond more properly to SIT (27).

Similarly, in the case of shrimp allergy, only three molecules can be evaluated (Tropomyosin, Arginine Kinase, and Sarcoplasmic $\mathrm{Ca}++$ Binding) out of about 14 distinct components currently identified, but not still available for diagnostic purposes (28). Therefore shrimp allergy diagnosis still represents a challenge for clinical allergologists.

In the case of seed storage protein, out of about 90 molecules registered in the IUIS/WHO database, only 13 components are available on the ISAC platform. Therefore, it is conceivable that in several cases a diagnostic approach based on the currently available molecules could not be sufficient for a comprehensive investigation (29). It is worth noting that Cor a 14 has been recently implemented in the most recent version of the ISAC (ISAC 112e), enhancing the diagnostic power of the test.

In conclusion, soon the recent European Union and Italian regulations in the matter of in vivo test could strongly impact on current diagnostic approach, increasing the usage of in vitro test in daily clinical practice. Multiplex testing is more than a promising tool for more precise and comprehensive profiling of allergic patients.

\section{Acknowledgements}

This project was funded in part by a research grant awarded by ThermoFisher Scientific, and Italian Ministry of Health (Ricerca Corrente).

\section{Conflict of interests}

Enrico SCALA has received consultant arrangements and speakers' bureau participation from Stallergenes and Thermo Fisher Scientific.

The rest of the authors declare that they have no conflict of interests. 


\section{Author contributions}

ES designed and completed the study, and GM organized and oversaw in vivo analysis. ES wrote the manuscript which was reviewed and amended by LC, IB, and DV.

\section{References}

1. Matricardi PM, Kleine-Tebbe J, Hoffmann HJ, et al. EAACI Molecular Allergology User's Guide. Pediatr Allergy Immunol 2016;27 Suppl 23:1-250.

2. Heinzerling L, Mari A, Bergmann KC, et al. The skin prick test --European standards. Clin Transl Allergy 2013;3(1):3.

3. Scala E, Alessandri C, Bernardi ML, et al. Cross-sectional survey on immunoglobulin E reactivity in 23077 subjects using an allergenic molecule-based microarray detection system. Clin Exp Allergy 2010;40(6):911-21.

4. Jensen-Jarolim E, Jensen AN, Canonica GW. Debates in allergy medicine: Molecular allergy diagnosis with ISAC will replace screenings by skin prick test in the future. World Allergy Organ J 2017;10(1):33.

5. Directive 2001/83/ec of the european parliament and of the council of 6 november 2001 on the community code relating to medicinal products for human use. Official Journal of the European Union 311, 67-128. 28-11-2004. Ref Type: Generic.

6. Larenas-Linnemann D, Luna-Pech JA, Mosges R. Debates in Allergy Medicine: Allergy skin testing cannot be replaced by molecular diagnosis in the near future. World Allergy Organ J 2017;10(1):32.

7. Mothes-Luksch N, Jordakieva G, Hinterholzl L, et al. Allergy diagnosis from symptoms to molecules, or from molecules to symptoms: a comparative clinical study. World Allergy Organ J 2018; 11(1):22.

8. Skypala IJ, Bull S, Deegan K, et al. The prevalence of pollen-food syndrome (PFS) and prevalence and characteristics of reported food allergy; a survey of UK adults aged 18-75 incorporating a validated PFS diagnostic questionnaire. Clin Exp Allergy 2013;43(8):928-40.

9. Asero R. Lipid transfer protein cross-reactivity assessed in vivo and in vitro in the office: pros and cons. J Investig Allergol Clin Immunol 2011;21(2):129-36.

10. Muraro A, Werfel T, Hoffmann-Sommergruber K, et al. EAACI food allergy and anaphylaxis guidelines: diagnosis and management of food allergy. Allergy 2014;69(8):1008-25.

11. Buzzulini F, Da RM, Scala E, et al. Evaluation of a new multiplex assay for allergy diagnosis. Clin Chim Acta 2019; 493:73-8.

12. Asero R, Mistrello G, Roncarolo D, Amato S. Parietaria Profilin Shows Only Limited Cross-Reactivity with Birch and Grass Profilins. Int Arch Allergy Immunol 2004;133(2):121-4.

13. Asero R, Mistrello G, Amato S. IgE Reactivity to Polcalcins Varies According to Pollen Source. J Investig Allergol Clin Immunol 2016;26(6):362-5.

14. Cabrera-Freitag P, Goikoetxea MJ, Beorlegui C, et al. Can component-based microarray replace fluorescent enzimoimmunoassay in the diagnosis of grass and cypress pollen allergy? Clin Exp Allergy 2011;41(10):1440-6.

15. Wohrl S, Vigl K, Zehetmayer S, et al. The performance of a component-based allergen-microarray in clinical practice. Allergy 2006;61(5):633-9.

16. Griffiths RLM, El-Shanawany T, Jolles SRA, et al. Comparison of the Performance of Skin Prick, ImmunoCAP, and ISAC Tests in the Diagnosis of Patients with Allergy. Int Arch Allergy Immunol 2017;172(4):215-23.

17. Heaps A, Carter S, Selwood C, et al. The utility of the ISAC Allergen Array in the investigation of Idiopathic Anaphylaxis. Clin Exp Immunol 2014;177(2):483-90.

18. Scala E, Cecchi L, Abeni D, et al. Pla a 2 and Pla a 3 reactivities identify plane tree-allergic patients with respiratory symptoms or food allergy. Allergy 2017;72(4):671-4.

19. Sanchez-Lopez J, Tordesillas L, Pascal M, et al. Role of Art v 3 in pollinosis of patients allergic to Pru p 3. J Allergy Clin Immunol 2014;133:1018-25.

20. Scala E, Till SJ, Asero R, et al. Lipid transfer protein sensitization: reactivity profiles and clinical risk assessment in an Italian cohort. Allergy 2015;70(8):933-43.

21. Borghesan F, Mistrello G, Amato S, Giuffrida MG, Villalta D, Asero R. Mugwort-fennel-allergy-syndrome associated with sensitization to an allergen homologous to Api g 5. Eur Ann Allergy Clin Immunol 2013;45(4):130-7.

22. Scala E, Abeni D, Cecchi L, et al. Molecular Recognition Profiles and Clinical Patterns of PR-10 Sensitization in a Birch-Free Mediterranean Area. Int Arch Allergy Immunol 2017;173(3):138-46.

23. Bobolea I, Barranco P, Pastor-Vargas C, Iraola V, Vivanco F, Quirce S. Arginine Kinase from the Cellar Spider (Holocnemus pluchei): A New Asthma-Causing Allergen. Int Arch Allergy Immunol 2011;155(2):180-6.

24. Celi G, Brusca I, Scala E, et al. House dust mite allergy in Italy-Diagnostic and clinical relevance of Der p 23 (and of minor allergens): A real-life, multicenter study. Allergy 2019;74(9):1787-9.

25. Brunetto B, Tinghino R, Braschi MC, Antonicelli L, Pini C, Iacovacci $P$. Characterization and comparison of commercially available mite extracts for in vivo diagnosis. Allergy 2010;65(2):184-90.

26. Casset A, Mari A, Purohit A, et al. Varying Allergen Composition and Content Affects the in vivo Allergenic Activity of Commercial Dermatophagoides pteronyssinus Extracts. Int Arch Allergy Immunol 2012;159(3):253-62.

27. Chen KW, Zieglmayer P, Zieglmayer R, et al. Selection of house dust mite-allergic patients by molecular diagnosis may enhance success of specific immunotherapy. J Allergy Clin Immunol 2019;143(3):1248-52.

28. Asero R, Scala E, Villalta D, et al. Shrimp Allergy: Analysis of Commercially Available Extracts for In Vivo Diagnosis. J Investig Allergol Clin Immunol 2017;27(3):175-82.

29. Scala E, Villalta D, Meneguzzi G, Giani M, Asero R. Storage molecules from tree nuts, seeds and legumes: relationships and amino acid identity among homologue molecules. Eur Ann Allergy Clin Immunol 2018; 50(4):148-55. 